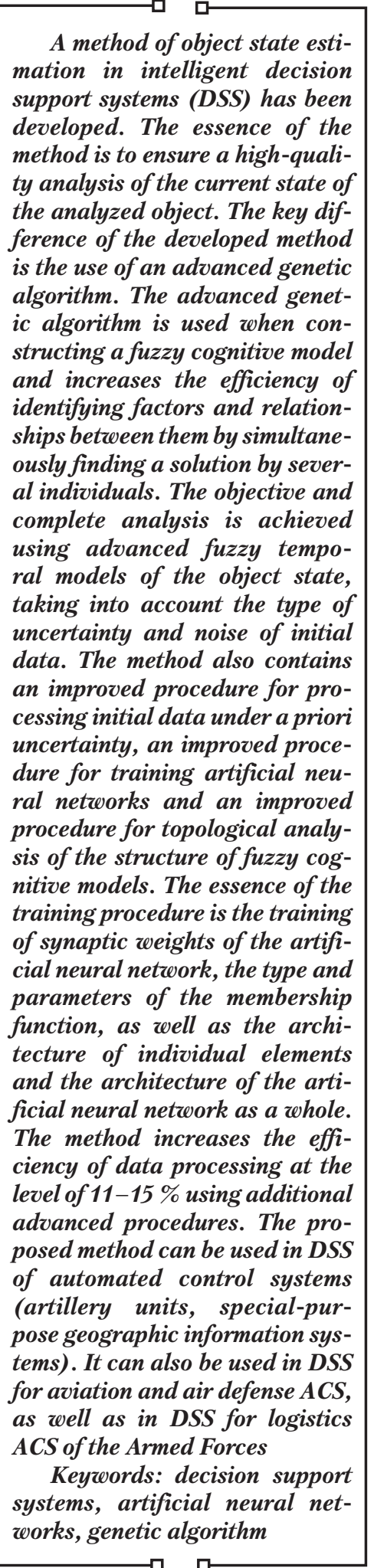

UDC 691.392

DOI: $10.15587 / 1729-4061.2021 .239854$

\section{DEVELOPMENT OF OBJECT STATE ESTIMATION METHOD IN INTELLIGENT DECISION SUPPORT SYSTEMS}

\author{
Vitali i Bezuhlyi \\ Corresponding author \\ Adjunct \\ Department of Management of Troops (Forces) in Peace* \\ E-mail: shturmanf7@gmail.com \\ Volodymy r O liynyk \\ Adjunct \\ Department of Airborne Troops and Special Forces* \\ I gor Romane n k o \\ Doctor of Technical Sciences, Professor, Leading Researcher \\ Scientific Research Department** \\ Oleksandr Zhuk \\ Doctor of Technical Sciences, Associate Professor, Head of Department \\ Department of Information Security in Telecommunication Systems and Networks*** \\ Vas y I K z a v kov \\ Doctor of Technical Sciences, Associate Professor, Head of Department \\ Department of Construction of Telecommunication Systems $* * *$ \\ Oleh Borysov \\ $\mathrm{PhD}$, Senior Lecturer \\ Department of Construction of Telecommunication Systems*** \\ Serhi K orobchenko \\ $\mathrm{PhD}$, Leading Researcher \\ Research Laboratory for Scientific and Methodological Support \\ of Military-Technical Cooperation** \\ Eduard Ost a p chuk \\ Deputy Head of Research Department \\ Research Department for the Development of Protection \\ and Survivability of Weapons and Military Equipment** \\ Taras Davydenko \\ Associate Professor \\ Department of Intelligence* \\ Andrii Shyshatskyi \\ $\mathrm{PhD}$, Senior Researcher \\ Research Department of Electronic Warfare Development** \\ *The National Defence University of Ukraine named after Ivan Cherniakhovsky \\ Povitroflotskyi ave., 28, Kyiv, Ukraine, 03049 \\ **Central Scientific Research Institute of Armament \\ and Military Equipment of the Armed Forces of Ukraine \\ Povitroflotskyi ave., 28, Kyiv, Ukraine, 03049 \\ ***Military Institute of Telecommunications and \\ Information Technologies named after Heroes of Kruty \\ Moskovska str., 45/1, Kyiv, Ukraine, 01011
}

\section{Introduction}

Decision support systems (DSS) are actively used in all spheres of human life. They are especially common in the processing of large data sets in databases, process development forecasting, providing informational and analytical support to the decision-making process of decision-makers. 
Existing DSS are based on statistical and artificial intelligence methods, which provide collection, processing, generalization of information about the object (process) state and forecasting of future state.

The creation of intelligent DSS has become a natural continuation of the widespread use of conventional DSS. The main fundamental difference between intelligent and DSS is the presence of feedback and adaptability to changes in input processes [1,2].

Intelligent DSS have been widely used to solve specific military tasks, namely [1, 2]:

- planning the deployment, operation of communication and data transmission systems;

- automation of troops and weapons control;

- training of personnel using training systems and complexes (training and monitoring the assimilation level of knowledge, skills and abilities by personnel);

- planning of combat training of units (subdivisions) and monitoring the quality of assimilation of learning material;

- reconnaissance of enemy objects and selection of fire destruction methods;

- determination of coefficients of operational and tactical importance of enemy objects;

- determination of the optimal composition of forces and means to achieve the goal of the operation (combat mission);

- collection, processing and generalization of intelligence information on the state of intelligence objects, etc.

The structure of intelligent DSS can be conventionally divided into 4 major layers:

- interface layer (interactivity and visualization);

- modeling layer (statistical models and machine learning; numerical models; game theory models, probability-statistical methods, etc.);

- data processing layer (data flow organization, work with databases and expert assessments);

- data collection layer (web scanning, sensors and programming interface).

Analysis of the experience of creating intelligent DSS shows that the most promising for their construction is an information technology based on a combination of different approaches [2-4]. One such approach is a combination of neuro-fuzzy cognitive models (NFCM), artificial neural networks (ANN) and genetic algorithms (GA). All this allows processing different types of data, adapting the structure to the type and amount of initial data, thereby increasing performance.

Evolutionary methods compared to conventional approaches have the following advantages:

- the ability to quickly adapt to the subject area, which, with little or no change, allows forming an NFCM and ANN structure that corresponds to a specific process;

- the ability to conduct a parallel search solution in several directions;

- avoiding the problem of falling into the local optimum trap;

- the ability to work under a priori uncertainty, nonlinearity, stochasticity, chaos and all kinds of disturbances;

- have both universal approximating properties and fuzzy inference capabilities.

Evolutionary methods are widely used to solve various problems of data mining, planning, control, identification, emulation, forecasting, intelligent control, etc. on each layer of intelligent DSS.
Despite a successful application to a wide range of data mining problems, these systems have a number of disadvantages.

The most significant ones are as follows:

1. The complexity of choosing system architecture. As a rule, the model based on the principles of computational intelligence has a fixed architecture. Therefore, adapting the system to new data that are different from previous data may be problematic.

2. Training in batch mode and training for several epochs requires significant time resources. Such systems are not adapted to work online with a fairly high rate of new data.

3. Problems in taking into account indicators that have a complex structure of relationships and contradict each other.

4. The difficulty of considering the indirect influence of interdependent components in conditions of uncertainty.

5. Nonlinear interaction of objects and processes, non-stochastic uncertainty, nonlinear interaction, partial inconsistency and significant interdependence of components.

Thus, the combination of NFCM and GA allows for the analysis (assessment) of the object state and ANN allows for deep learning of databases.

There is an urgent scientific task to develop an estimation method in intelligent decision support systems using NFCM, GA and ANN.

\section{Literature review and problem statement}

The work [9] presents a cognitive modeling algorithm. The main advantages of cognitive tools are determined. When constructing the experimental model, target factors of the cognitive map were determined, connectivity analysis was performed, and the process of perturbation propagation on the graph was studied. The proposed model is used to assess and forecast economic activities and determine expected values of parameters that need to be monitored to diagnose trends in industrial development. The disadvantages of this approach include the lack of consideration of the type of uncertainty about the state of the analysis object.

The work [10] reveals the essence of cognitive modeling and scenario planning. The system of complementary principles of constructing and implementing scenarios is proposed, various approaches to constructing scenarios are allocated, the procedure for modeling scenarios on the basis of fuzzy cognitive maps is described. It is proposed to identify the concepts of the cognitive map by analyzing the internal and external environments of the organization, which will allow you to systematically look at the business conditions of the enterprise, predict further development and make right management decisions. The approach proposed by the authors does not take into account the type of uncertainty about the state of the analysis object and the delay in processing object state data.

The work [11] carried out the analysis of the main approaches to cognitive modeling. Cognitive analysis allows you to explore problems with fuzzy factors and relationships, take into account changes in the external environment and take advantage of objective trends in the situation. It is necessary to develop a system of criteria for the formalization and automation of decision-making in problem areas. It is also stated that the objectivity of the information processed must be taken into account. 
The work [12] presents the method of large data sets analysis. This method is focused on finding hidden information in large data sets. The method involves the operations of generating analytical baselines, reducing variables, identifying sparse features and specifying rules. The disadvantages of this method include the inability to take into account different decision evaluation strategies.

The work [13] gives the mechanism of transformation of information models of construction objects to their equivalent structural models. This mechanism is designed to automate the necessary transformation, modification and addition operations during such information exchange. The disadvantages of this approach include the inability to assess the adequacy and reliability of the information transformation process.

The work [14] carried out the development of an analytical web platform for studying the geographical and temporal distribution of incidents. The web platform, contains several information panels with statistically significant results by territory. The web platform includes certain external sources of data on social and economic issues, which allow studying the relationship between these factors and the distribution of incidents at different geographical levels. The disadvantages of this analytical platform include the inability to assess the adequacy and reliability of the information transformation process, as well as high computational complexity.

The work [15] developed a method of fuzzy hierarchical assessment of the library services quality. This method allows evaluating the quality of libraries by a set of input parameters. The disadvantages of this method include the inability to assess the adequacy and reliability of the assessment.

The work [16] performed an analysis of 30 algorithms for processing large data sets. Their advantages and disadvantages are shown. It is found that the analysis of large data sets should be performed in layers, in real time and be able to self-study. The disadvantages of these methods include high computational complexity and the inability to verify the adequacy of the estimates.

The work [17] presents the approach of estimation of input data for decision - support systems. The essence of the proposed approach is to cluster the basic set of input data, analyze them, and train the system on the basis of the analysis. The disadvantages of this approach are the gradual accumulation of assessment and training errors due to the inability to assess the adequacy of decisions.

The work [18] presents the approach to processing data from different sources of information. The disadvantages of this approach include the low accuracy of the assessment and the inability to verify the reliability of the assessment.

The work [19] carried out a comparative analysis of existing decision support technologies, namely: analytic hierarchy process, neural networks, fuzzy set theory, genetic algorithms and neuro-fuzzy modeling. The advantages and disadvantages of these approaches are indicated. The scope of their application is defined. The analytic hierarchy process has been shown to work well with complete initial data, but it has a high degree of subjectivity due to the need for experts to compare alternatives and select evaluation criteria. The use of fuzzy set theory and neural networks is justified for forecasting problems in conditions of risk and uncertainty.

The work [20] developed a method of structural-target analysis of the development of poorly structured systems. The approach to studying conflict situations caused by contradictions in the interests of subjects influencing the development of the investigated system and methods for solving weakly structured problems based on the formation of situation development scenarios is proposed. The problem is defined as a mismatch of the existing system state to the required one, which is set by the subject of management. However, the disadvantages of the proposed method include the problem of local optimum and the impossibility of conducting a parallel search.

The work [21] presents a cognitive approach to the simulation of complex systems. The advantages of this approach, which allows describing the hierarchical composition of the system, are shown. The disadvantages of the proposed approach include the one-way search for solutions.

The analysis of the works [9-21] showed the following known approaches based on general scientific methods: system, comparative, structural-functional analysis, method of expert assessments, method of scenario analysis of socio-economic systems and theoretical-information approach. Common limitations of the existing methods of multi-criteria fuzzy evaluation of alternatives are:

- the complexity of forming a multilevel evaluation structure;

- the lack of consideration of the compatibility of unevenly significant indicators;

- the inability to jointly perform direct and inverse evaluation tasks supported by choosing the best solutions;

- the impossibility of simultaneous search for solutions in different directions;

- getting of local optimum into the problem;

- failure to take into account the type of uncertainty of initial object state data and data noise.

To this aim, it is proposed to develop an estimation method in intelligent decision - support systems based on NFCM, $\mathrm{ANN}$ and GA.

\section{The aim and objectives of the study}

The aim of the study is to develop a method of object state estimation in intelligent decision support systems, which would allow for the analysis of the object state.

To achieve the aim, the following objectives were set:

- to formulate a mathematical description of the estimation process in intelligent decision support systems;

- to determine the algorithm for implementing the method;

- to give an example of application of the proposed method in the analysis of the operational situation of the troops (forces) group.

\section{Research materials and methods}

The object of research is military monitoring objects. The objects can be control points of different control units containing a set of various control and data transmission means, air observation means, reconnaissance means, etc. The main hypothesis of the research is to determine the affiliation of the monitoring object (determination of its origin and quantitative and qualitative composition). Control points of individual brigades, operational and tactical groups of troops, operational groups of troops (forces) are considered as monitoring objects.

The research used the general provisions of artificial intelligence theory to solve the problem of object state anal- 
ysis in intelligent decision support systems. Thus, artificial intelligence theory is the basis of the research.

Fuzzy cognitive models were used to solve the problem of describing the subsequent state of dynamic objects. They describe how complex multi-level objects change over time. An advanced genetic algorithm is used to increase the efficiency of constructing a fuzzy cognitive model. The research also uses the method of training artificial neural networks developed in previous works, which allows for deep learning of artificial neural networks. The essence of deep learning is to train the architecture, type and parameters of the membership function. The simulation was performed using MathCad 2014 software (USA) and Intel Core i3 PC (USA).

\section{Results of research on estimation method development}

5. 1. Mathematical description of the estimation process in intelligent decision support systems

A mathematical description of the process of object state estimation for a dynamic system can be presented as:

$$
\begin{aligned}
& \forall t \in\{1, \ldots, T, \ldots\} S_{t}=
\end{aligned}
$$

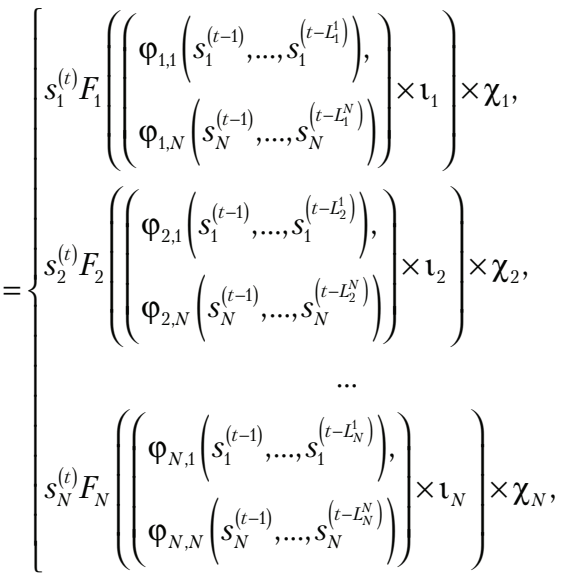

where $S$ is the multidimensional time series; $S_{t}=\left(s_{1}^{(t)}, s_{2}^{(t)}, \ldots, s_{N}^{(t)}\right)$ is the time sample of the state of the analysis object presented as a multidimensional time series at the $t$-th time point; $s_{j}^{(t)}$ is the value of the $j$-th component of the multidimensional time series at the $t$-th time point; $L_{j}^{i}$ is the maximum value of the time delay of the $i$-th component relative to the $j$-th one; $\varphi_{i j}$ is the operator to account for the interaction between the $i$-th and $j$-th components of the multidimensional time series; $F_{i}$ is the conversion to obtain $s^{(t)}, i=1, \ldots, N ; N$ is the number of components of the multidimensional time series; $\mathrm{t}$ is the operator to account for the degree of object state awareness; $\chi$ is the operator to account for the noise degree of object state data.

From expression (2), we can conclude that it allows describing processes in the analysis object, taking into account time delays. Delays are required to collect, process and summarize information, taking into account the degree of object state awareness and data noise.

\section{2. Algorithm for implementing the estimation meth-} od in intelligent decision support systems

The estimation method in intelligent decision support systems consists of the following steps (Fig. 1). It should be noted that the main procedures of the proposed method are described in detail in [23]. However, the key difference between this method and the known ones is that step 3 of this method uses the genetic algorithm improved by the authors, the main stages of which are shown in Fig. 2. The genetic algorithm allows for a multi-directional search by several individuals when constructing a fuzzy cognitive model.

1. Entering initial data (step 1 in Fig. 1).

At this stage, initial available data about the object to be analyzed are entered.

2. Processing initial data taking into account uncertainty (step 2 in Fig. 1).

At this stage, the type of uncertainty about the analysis object state is taken into account and the basic object state model is initialized [22].

3. Setting the values of factors and relationships between them (step 3 in Fig. 1).

At this stage, using the improved genetic algorithm, a parallel search for a solution is performed simultaneously by several individuals of the population to determine factors and relationships between them.

The idea of estimation using a genetic algorithm is to analyze possible object state estimation alternatives [9]. The estimation of each $k$-th object state alternative is carried out on the basis of the found expected alternative $s\left(x^{(k)}\right), k=1, \ldots, m$, and formation of an interval multicriteria estimate $P\left(x^{(k)}\right)$. Here are the assumptions:

- firstly, four object state alternatives $\left(x^{(1)}, x^{(2)}, x^{(3)}, x^{(4)}\right)$ are developed and analyzed;

- secondly, four partial criteria of the state of the analysis object are considered, which can be represented as fuzzy trapezoidal numbers, and their boundaries may intersect. If it is necessary to increase the number of possible object state gradations, fuzzy polyhedral numbers can be used, which are presented in $[22,23]$;

- thirdly, the partial state criterion is denoted by $s\left(x^{(k)}\right)$, $s\left(x^{(k)}\right)=\left(s_{1}^{(k)}, s_{2}^{(k)}, s_{3}^{(k)}, s_{4}^{(k)}\right)\left(s_{1}^{(k)}, s_{4}^{(k)}\right.$ are the pessimistic and optimistic estimates of the object state; $\left[s_{2}^{(k)}, s_{3}^{(k)}\right]$ is the interval of the expected state of the analysis object).

There is a limited set of object state alternatives $X=$ $=\left\{x^{(1)}, x^{(2)}, \ldots, x^{(m)}\right\}$, where each alternative is evaluated by a tuple of $n$ non-standardized interval partial criteria $C=\left\langle c_{j}\left(x^{(k)}\right)\right\rangle, j=\overline{1, n}$. The task is to find the vector of the expected state of the analysis object for each $k$-th object state alternative:

$$
s\left(x^{(k)}\right)=\left(s_{1}^{(k)}, s_{2}^{(k)}, s_{3}^{(k)}, s_{4}^{(k)}\right), \quad k=\overline{1, m}
$$

regarding the assessment of generalized utility characterizing the implementation of each $k$-th object state alternative:

$$
P\left(x^{k}\right)=\sum_{j=1}^{n} w_{j}^{\mathrm{norm}} p_{j}^{\mathrm{norm}}\left(x^{(k)}\right), \quad k=\overline{1, m}, \quad j=\overline{1, n},
$$

with the possibility of further selection of an acceptable alternative, $w_{j}^{\text {norm }}$ is the normalized interval coefficient of relative importance of the $j$-th partial criterion of alternatives $x^{(k)} \in X, \quad p_{j}^{\text {norm }}\left(x^{(k)}\right)$ is the normalized interval partial criteria of alternatives $x^{(k)} \in X\left(0<p_{j}^{\text {norm }}\left(x^{(k)}\right)<1\right)$.

Thus, the task of evaluating the feasibility of object state alternatives is described by the following objective function:

$$
P\left(x^{(k)}\right)=\sum_{j=1}^{n} w_{j}^{\text {norm }} p_{j}^{\text {norm }}\left(x^{(k)}\right) \rightarrow \max , x^{(k)} \in X,
$$

and constraints 


$$
\begin{aligned}
& w_{j}^{\text {norm }}=\left[\alpha_{j 1}, \alpha_{j 2}, \alpha_{j 3}, \alpha_{j 4}\right], \\
& s_{\min } \leq s\left(x^{(k)}\right) \leq s_{\max },
\end{aligned}
$$

where $\sum_{j=1}^{n} \alpha_{j 1}<1, \quad \sum_{j=1}^{n} \alpha_{j 4}>1 ; s_{1}$ and $s_{2}$ are the values of the minimum and maximum alternatives. The solution of the problem is combinatorial in nature and can be formulated as a search for the best solution. To find the expected alternative $s\left(x^{(k)}\right)$, a genetic algorithm is proposed, consisting of the following steps (Fig. 2).

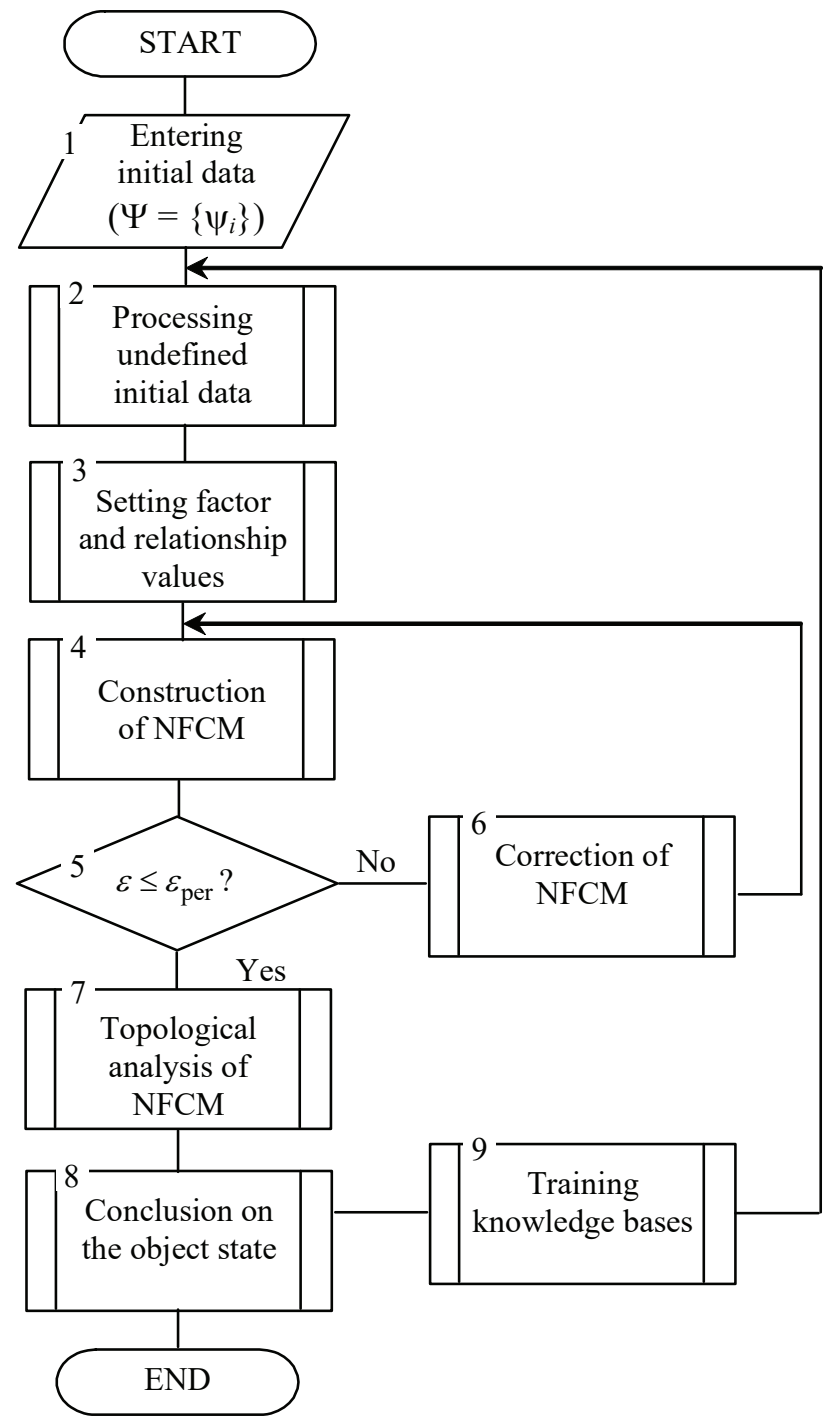

Fig. 1. Algorithm of the object state analysis method

Step 3. 1. Entering initial data: $s\left(x^{(k)}\right)$ is the unregulated interval partial criteria of alternatives; $s_{\min }$ and $s_{\max }$ are the values of minimum and maximum alternatives; $k o l$ is the number of iterations; $d$ is the crossing over position; $q$ is the mutation position; $w_{j}$ is the interval coefficient of relative importance of the $j$-th partial criterion of alternatives, as well as the determination of the initial population size $P=2 p$.

Step 3. 2. Determination of necessary optimization conditions: objective function (3) and constraints (4), (5).

Step 3.3. Formation of the initial population - parent individuals (four options of object state vectors for each $k$-th object state alternative) using a random number generator:

$$
\begin{aligned}
s_{1}^{P}\left(x^{(1)}\right) & =\left(s_{1}^{P(1)}, s_{2}^{P(1)}, s_{3}^{P(1)}, s_{4}^{P(1)}\right), \\
s_{2}^{P}\left(x^{(2)}\right) & =\left(s_{1}^{P(2)}, s_{2}^{P(2)}, s_{3}^{P(2)}, s_{4}^{P(2)}\right), \\
s_{3}^{P}\left(x^{(3)}\right) & =\left(s_{1}^{P(3)}, s_{2}^{P(3)}, s_{3}^{P(3)}, s_{4}^{P(3)}\right), \\
s_{4}^{P}\left(x^{(4)}\right) & =\left(s_{1}^{P(4)}, s_{2}^{P(4)}, s_{3}^{P(4)}, s_{4}^{P(4)}\right) .
\end{aligned}
$$

Formation of the initial population in which chromosomes are encoded as 8-digit sets of values. This type of chromosome allows leaving the genes arranged sequentially after the application of the mutation operator. Preservation of the continuous arrangement of genes allows the genetic crossing operator to copy continuous sections of the random access memory of the parent chromosomes into the daughter chromosome, which accelerates the crossing [10].

Step 3. 4. Checking the condition is met. If condition (5) for parent individuals is met, go to step 8; otherwise, go to step 3 .

Step 3.5. Calculation of resource capacity of the obtained values and checking conditions for non-exceeding $r e s_{\text {add }}$ for each individual in the initial population:

$$
\operatorname{res}_{i}=f\left(U_{e f f}\right),
$$

where $U_{\text {eff }}$ is the key efficiency indicator.

Step 3. 6. Calculation of the membership function of the level of target achievement $\Lambda_{i j}^{\mathrm{targ}}$, which consists in the implementation of the iterative procedure for recalculating target indicators based on the developed fuzzy cognitive model:

$$
\Lambda_{i j}^{\mathrm{targ}}=f\left(U_{e f f}\right), \quad j=\overline{1, k}
$$

Step 3. 7. Calculation of the stop parameter of the algorithm based on the minimum deviation of the level of target achievement relative to the required value:

$$
\begin{aligned}
& \Delta \Lambda_{i j}^{\mathrm{targ}}=\Lambda_{i j}^{\mathrm{targ}}-\Lambda_{i \text { need }}^{\mathrm{targ}}, \\
& \Delta \Lambda=\min _{i} \min _{j} \Delta \Lambda_{i j}^{\mathrm{targ}} .
\end{aligned}
$$

Step 3. 8. Adding the vector $s_{k}^{P}\left(x^{(k)}\right)$ to the population.

Step 3. 9. Implementation of a single-point crossover operation with the obtained options of the vectors of the $k$-th alternative $s^{P}\left(x^{(k)}\right)$ (crossover point $d<4$ ). Selection of individuals and formation of pairs for crossing in accordance with their fitness function. The proposed selection procedure is implemented using the mechanism of adaptation of the optimization algorithm parameters described in [1].

This adaptation mechanism is based on a compromise between the convergence rate and the quality of the obtained local-optimum solution, its essence is that the probability of selecting individuals varies flexibly depending on the search history [11].

For this purpose, the normal law of selection probability distribution is used. The mathematical expectation is taken equal to the value of the best fitness function for a given generation of the population chromosome. If in the next generation, there was a change in the best chromosome, the variance takes the maximum value, thereby expanding the search range. If for several generations, the best chromosome is found, the variance decreases, in the simplest case, it is proportional to the number of generations:

$$
D=D_{\max }-\beta \times g
$$


where $D_{\max }$ is the maximum value of the variance; $\beta$ is the coefficient that determines the convergence rate of the algorithm; $g$ is the number of "unsuccessful" generations.

The mathematical expectation of the distribution function is equal to the value of the function $F_{\max }$. The random variable $X_{i}$ is continuous, unlike the discrete values $F_{k}$, $k=1,2, \ldots, M$, and it is necessary to choose the value $F_{k}$ of the fitness function, the distance from which to $F_{\max }$ would be closest to the distance from $X_{i}$ to $F_{\max }$ :

$$
k=\arg \min \left\|F_{\max }-X|-| F_{\text {max }}-X\right\| .
$$

In this case, this value will be $F_{i}$.

In subsequent selection cycles, consideration should be given to the fitness function values corresponding to the individuals already selected in the previous cycles. This will ensure the diversity of the population.

The described mechanism forms prerequisites for elite selection, preserves the best of the found chromosomes of the population, and is used in three cases:

- before the crossover stage to select crossing individuals:

- before the mutation stage to select mutating individuals;

- after the application of all GA operators to select the most suitable individuals for the next generation.
As a result of the crossover operation, 12 descendant individuals are obtained:

$$
\begin{aligned}
& s_{1}^{P}\left(x^{(1)}\right)=\left(s_{1}^{P(1)}, s_{d}^{P(1)}, s_{d+1}^{P(2)}, s_{4}^{P(2)}\right), \\
& s_{2}^{P}\left(x^{(2)}\right)=\left(s_{1}^{P(2)}, s_{d}^{P(2)}, s_{d+1}^{P(1)}, s_{4}^{P(1)}\right), \\
& s_{3}^{P}\left(x^{(1)}\right)=\left(s_{1}^{P(1)}, s_{d}^{P(1)}, s_{d+1}^{P(3)}, s_{4}^{P(3)}\right), \\
& s_{4}^{P}\left(x^{(3)}\right)=\left(s_{1}^{P(3)}, s_{d}^{P(3)}, s_{d+1}^{P(1)}, s_{4}^{P(1)}\right), \\
& s_{5}^{P}\left(x^{(1)}\right)=\left(s_{1}^{P(1)}, s_{d}^{P(1)}, s_{d+1}^{P(4)}, s_{4}^{P(4)}\right), \\
& s_{6}^{P}\left(x^{(4)}\right)=\left(s_{1}^{P(4)}, s_{d}^{P(4)}, s_{d+1}^{P(1)}, s_{4}^{P(1)}\right), \\
& s_{7}^{P}\left(x^{(2)}\right)=\left(s_{1}^{P(2)}, s_{d}^{P(2)}, s_{d+1}^{P(3)}, s_{4}^{P(3)}\right), \\
& s_{8}^{P}\left(x^{(3)}\right)=\left(s_{1}^{P(3)}, s_{d}^{P(3)}, s_{d+1}^{P(2)}, s_{4}^{P(2)}\right), \\
& s_{9}^{P}\left(x^{(2)}\right)=\left(s_{1}^{P(2)}, s_{d}^{P(2)}, s_{d+1}^{P(4)}, s_{4}^{P(4)}\right), \\
& s_{10}^{P}\left(x^{(4)}\right)=\left(s_{1}^{P(4)}, s_{d}^{P(4)}, s_{d+1}^{P(2)}, s_{4}^{P(2)}\right), \\
& s_{11}^{P}\left(x^{(3)}\right)=\left(s_{1}^{P(3)}, s_{d}^{P(3)}, s_{d+1}^{P(4)}, s_{4}^{P(4)}\right), \\
& s_{12}^{P}\left(x^{(4)}\right)=\left(s_{1}^{P(4)}, s_{d}^{P(4)}, s_{d+1}^{P(3)}, s_{4}^{P(3)}\right) .
\end{aligned}
$$

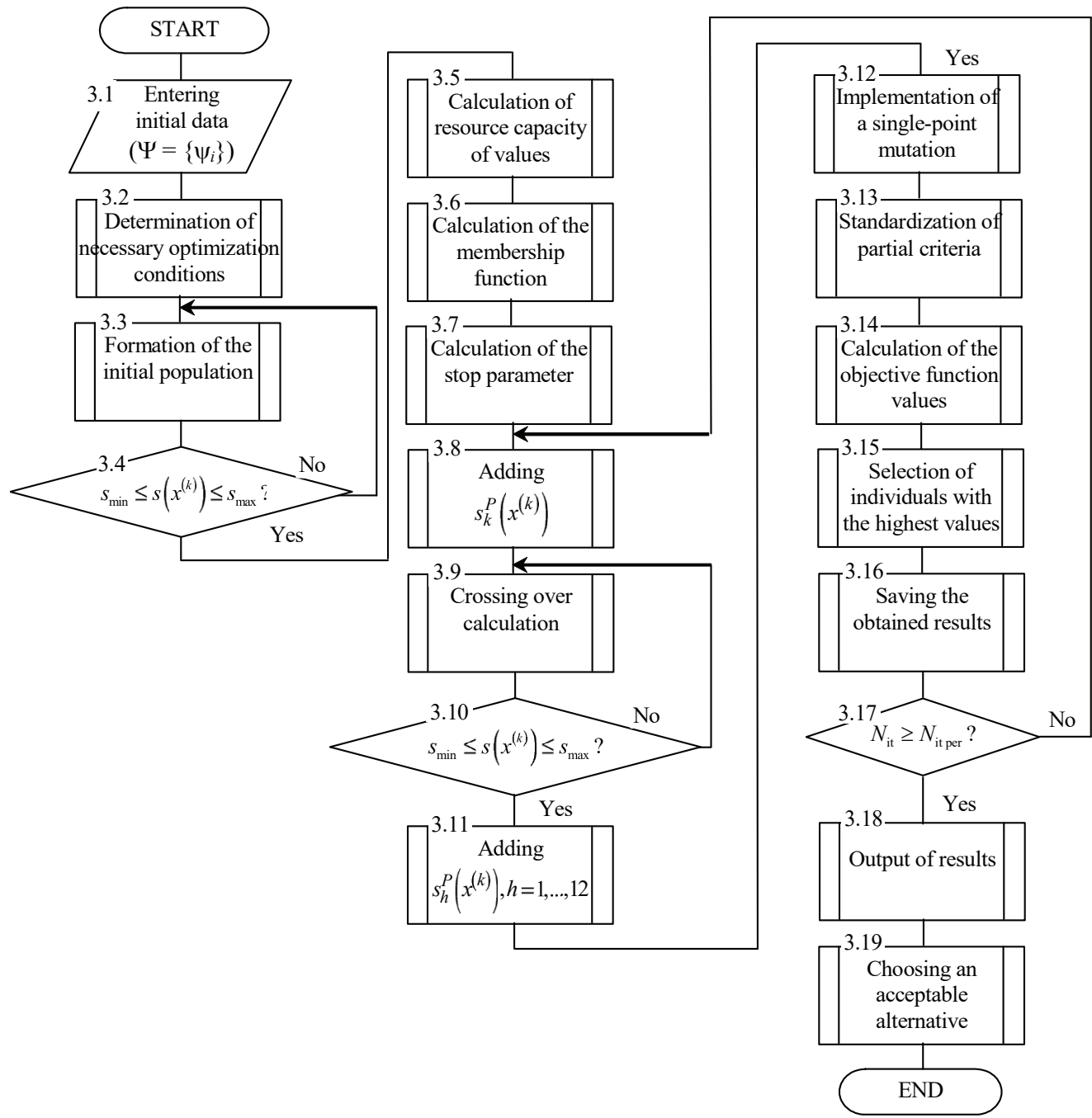

Fig. 2. Sequence of execution of the advanced genetic algorithm 
The crossing over (crossover, crossing) operator allows creating descendant chromosomes on the basis of crossing parent chromosomes. Single-point crossing is the cutting of parent chromosomes at a random common cut (break) point and the exchange of the right parts ("tails" of the chromosomes).

Step 3. 10. Checking the condition is met. If condition (5) for the obtained descendant individuals is met, go to step 11; otherwise, go to step 9.

Step 3. 11. Adding the vector $s_{h}^{P}\left(x^{(k)}\right), \quad h=1, \ldots, 12$ to the population.

Step 3.12. Implementation of the operation of single-point mutation of the obtained 12 descendant individuals (mutation point $q<4$ ). With the arrival of the descendant individual, a new descendant individual with mutated genes $\bar{s}_{1}^{-M}\left(x^{(1)}\right)=\left(s_{1}^{M}, s_{q}^{M},{ }^{-M}{ }_{q+1}, s_{4}^{M}\right)$ is obtained by mutation. Similarly, this operation is performed with other descendant individuals. The mutation operator allows making random changes in individuals, which subsequently acquire new properties. Single-point mutation is a random selection of a gene that exchanges its value with a nearby gene.

Step 3. 13. Implementation of rationing of partial criteria $p_{j}^{\text {norm }}\left(x^{(k)}\right)$, including the expected cost of alternatives for each $k$-th alternative of object state estimation by the formula

$$
\begin{aligned}
& c_{j}\left(x^{(k)}\right)=\left[c_{j 1}\left(x^{(k)}\right), c_{j 2}\left(x^{(k)}\right), c_{j 3}\left(x^{(k)}\right), c_{j 4}\left(x^{(k)}\right)\right], \\
& p_{j}^{\operatorname{norm}}\left(x^{(k)}\right)=\left[\frac{c_{j 1}\left(x^{(k)}\right)}{c_{j}^{\max }}, \frac{c_{j 2}\left(x^{(k)}\right)}{c_{j}^{\max }}, \frac{c_{j 3}\left(x^{(k)}\right)}{c_{j}^{\max }}, \frac{c_{j 4}\left(x^{(k)}\right)}{c_{j}^{\max }}\right], \\
& c_{j}^{\max }=\max _{1 \leq k \leq m}\left\{c_{j 4}^{(k)}\right\},
\end{aligned}
$$

where $c_{j 1}\left(x^{(k)}\right), c_{j 4}\left(x^{(k)}\right)$ are the pessimistic and optimistic estimates of the interval boundaries, $c_{j 2}\left(x^{(k)}\right), c_{j 3}\left(x^{(k)}\right)$ is the range of the most expected values. Calculation of the interval coefficients of relative importance $w_{j}^{\text {norm }}$ norm of partial criteria by the formula:

$$
w_{j}^{\text {norm }}=\frac{w_{j}}{\sum_{j=1}^{n} w_{j}}, j=\overline{1, n},
$$

where $w_{j}$ is the interval coefficient of relative importance of the $j$-th fraction of the criterion, which can be represented as intervals, fuzzy triangular, trapezoidal and polyhedral numbers.

Step 3. 14. Calculation of the values of the objective function (2) for all individuals of the population, namely for all options of the vectors of the expected object state.

Step 3. 15. Selection of four individuals (four vectors) among 12 possible with the largest values of the objective function, which will be the parents for the next iteration (generation), or in the case of all iterations - the result of calculations.

Since the proposed task considers four alternatives of object state estimation, only four vectors of the expected state are selected. The selection of individuals is made by the rank method, so individuals of the population are ranked according to the value of their fitness function (ranking is carried out by increasing values). Since the estimates of the generalized utility $P\left(x^{(k)}\right)$ are presented as trapezoidal fuzzy numbers, the Chu-Park method is used to select an acceptable object state alternative.
Step 3. 16. Saving the obtained results.

Step 3. 17. Checking the condition is met. If the condition of execution $N_{\text {it }} \geq N_{\text {it add }}$ iterations is met, go to step 18; otherwise, go to step 8 .

Step 3.18. Output of results (vectors of alternatives $s\left(x^{(k)}\right)$ and multicriteria estimate $\mathrm{Cp}\left(P\left(x^{(k)}\right)\right)$ for each $k$-th alternative).

Step 3. 19. Choosing an acceptable alternative. An acceptable alternative is one in which the generalized utility is the greatest.

End of the algorithm.

Procedures 4-9 of the proposed method are described in detail in [24].

5. 3. Example of using the proposed method to analyze the operational situation of the troops (forces) group

Simulation of the solution search method according to the algorithm in Fig. 2 and expressions (1)-(13) is carried out. The simulation of the proposed evaluation method is performed in the MathCad 14 software environment (USA). The task of the simulation,was to assess the elements of the operational situation of the troops (forces) group.

Initial data for assessing the state of the operational situation using the proposed method:

- the number of object state data sources - 3 (radio monitoring devices, remote earth sensing devices and unmanned aerial vehicles). To simplify the simulation, the same amount of each device was taken -4 devices;

- the number of information features that determine the state of the monitoring object -12 . These parameters include: affiliation, type of organizational-staff formation, priority, minimum width on the front, maximum width on the front. The number of personnel, the minimum depth on the flank, the maximum depth on the flank, the total number of personnel, the number of weapons samples, the number of types of weapons samples and the number of communication devices are also taken into account;

- options of organizational-staff formation - company, battalion, brigade.

The cognitive map of the operational environment of the group is a square table (incidence matrix). The rows and columns mutually uniquely correspond to the basic factors describing the object under study, and the number at the intersection of the $i$-th row and the $j$-th column describes the effect of the $i$-th factor on the $j$-th factor (Table 1).

Table 1

Incidence matrix of the cognitive map of situation assessment

\begin{tabular}{|c|c|c|c|c|c|c|c|c|c|c|c|c|}
\hline No. & $w_{1}$ & $w_{2}$ & $w_{3}$ & $w_{4}$ & $w_{5}$ & $w_{6}$ & $w_{7}$ & $w_{8}$ & $w_{9}$ & $w_{10}$ & $w_{11}$ & $w_{12}$ \\
\hline$w_{1}$ & 0 & 1 & 1 & 0 & 0 & 0 & 0 & 1 & 0 & 1 & 1 & 0 \\
\hline$w_{2}$ & 0 & 0 & 1 & 0 & 1 & 1 & 1 & 0 & 0 & 1 & 1 & 0 \\
\hline$w_{3}$ & 0 & 1 & 0 & 0 & 1 & 0 & 0 & -1 & 0 & 1 & 0 & -1 \\
\hline$w_{4}$ & 0 & 0 & 1 & 0 & 0 & 1 & -1 & 0 & 0 & 1 & 1 & 0 \\
\hline$w_{5}$ & 0 & 1 & 1 & 0 & 0 & 0 & 0 & 1 & 1 & 1 & 1 & 0 \\
\hline$w_{6}$ & 0 & 1 & 0 & 0 & -1 & 0 & 1 & 1 & -1 & 1 & 1 & 0 \\
\hline$w_{7}$ & 1 & -1 & 1 & 0 & 0 & -1 & 0 & 1 & 0 & 1 & 0 & 0 \\
\hline$w_{8}$ & 0 & -1 & 1 & 1 & 1 & -1 & 0 & 0 & 0 & 0 & 0 & 0 \\
\hline$w_{9}$ & 1 & 0 & 1 & 1 & -1 & 1 & 1 & 0 & 0 & 1 & 1 & 0 \\
\hline$w_{10}$ & 1 & -1 & 0 & 1 & 0 & 1 & 0 & -1 & 0 & 0 & 0 & 0 \\
\hline$w_{11}$ & 1 & 1 & 1 & -1 & 0 & 1 & 0 & 0 & 0 & 1 & 1 & 1 \\
\hline$w_{12}$ & 0 & 0 & 1 & 1 & 0 & 1 & 1 & 1 & 1 & 1 & 0 & 0 \\
\hline
\end{tabular}


Tables 2, 3 present comparative results of evaluating the training efficiency of evolving artificial neural networks.

Before training, the observation features were normalized at the interval $[0,1]$.

Table 2

Comparative results of evaluating the training efficiency of evolving artificial neural networks

\begin{tabular}{|c|c|c|c|}
\hline System & $\begin{array}{c}\text { Algorithm } \\
\text { parameters }\end{array}$ & $\begin{array}{c}\text { XB (Xie- } \\
\text { Beni Index) }\end{array}$ & $\begin{array}{c}\text { Time, } \\
\text { s }\end{array}$ \\
\hline FCM (Fuzzy C-Means) & - & 0.1903 & 2.69 \\
\hline EFCM & Dthr $=0.24$ & 0.1136 & 0.14 \\
\hline EFCM & Dthr $=0.19$ & 0.1548 & 0.19 \\
\hline Proposed system (batch mode) & delta $=0.1$ & 0.0978 & 0.37 \\
\hline Proposed system (online mode) & delta $=0.1$ & 0.1127 & 0.25 \\
\hline
\end{tabular}

Table 3

Comparative results of clustering

\begin{tabular}{|c|c|c|c|}
\hline System & $\begin{array}{c}\text { Algorithm } \\
\text { parameters }\end{array}$ & $\begin{array}{c}\text { XB (Xie- } \\
\text { Beni Index) }\end{array}$ & $\begin{array}{c}\text { Time, } \\
\mathrm{s}\end{array}$ \\
\hline FCM (Fuzzy C-Means) & Dthr $=0.6$ & 0.2963 & 0.81 \\
\hline EFCM & Dthr $=0.6$ & 0.2330 & 0.54 \\
\hline Proposed system (batch mode) & delta $=0.4$ & 0.2078 & 0.45 \\
\hline Proposed system (online mode) & delta $=0.4$ & 0.2200 & 0.30 \\
\hline
\end{tabular}

It is worth noting that the proposed training procedure showed a better PC (partition coefficient, PC) result compared to EFCM and a better operating time result compared to FCM. The research showed that this training procedure provides an average of 11-15\% higher training efficiency of artificial neural networks and does not accumulate errors during training (Tables 2, 3).

This can be seen from the results in the last rows of Table 2, 3, as the difference of the Xie-Beni index. However, as already mentioned, the known methods accumulate errors, so the proposed method suggests the use of evolving artificial neural networks.

Consider the genetic algorithm described above on a specific example.

There are four object state alternatives $\left(x_{1}^{(k)}, x_{2}^{(k)}, x_{3}^{(k)}, x_{4}^{(k)}\right)$, described by four partial criteria. The criteria are: $c_{1}$ - duration of the operation, days; $c_{2}-$ number of personnel, people; $c_{3}$ - probability of achieving the goal of the operation, may not be achieved in whole or in part; $c_{4}-$ cost of the operation $s\left(x_{i}\right)=[767,830] \mathrm{CU},(i=1 \ldots 4)$. It is necessary to assess the state of the object and then select its current state.

The estimated values of the partial criteria of the object state for each alternative are presented in Table 4.

Based on the approaches given in [2,4], the coefficients of relative importance of the partial criteria in the form of trapezoidal numbers are obtained:

$$
\begin{aligned}
& w_{1}=[3,3.4,3.7,4.3], w_{2}=[3.2,3.65,3.76,4.2], \\
& w_{3}=[2.2,2.6,2.77,3.12], w_{4}=[2.5,2.85,3.3,3.4],
\end{aligned}
$$

where $w_{4(s)}$ is the weighting factor of relative importance of the criterion of the cost of the operation of the troops (forces) group.

The initial values of the genetic algorithm parameters are as follows: crossover point (crossing) - 1; mutation point - 1; number of iterations -10 .
Table 4

Estimated values of the partial criteria of the object state for each current state alternative

\begin{tabular}{|c|c|c|c|}
\hline \multirow{2}{*}{$\begin{array}{c}\text { Alternatives to achieving } \\
\text { the goal of the operation }\end{array}$} & \multicolumn{3}{|c|}{ Partial object state criteria } \\
\cline { 2 - 4 } & $c_{1}$ & $c_{2}$ & $c_{3}$ \\
\hline \multirow{2}{*}{$x^{(1)}$} & {$[9.5,10$,} & {$[10,12$,} & {$[0.8,0.82$,} \\
& $10.7,11]$ & $13,15]$ & $0.83,0.84]$ \\
\hline \multirow{2}{*}{$x^{(2)}$} & {$[8.7,9$,} & {$[8,9$,} & {$[0.83,0.85$,} \\
& $9.5,10]$ & $10,12]$ & $0.86,0.87]$ \\
\hline \multirow{2}{*}{$x^{(3)}$} & {$[9.2,9.6$,} & {$[9,10$,} & {$[0.8,0.83$,} \\
& $10,10.3]$ & $11,12]$ & $0.84,0.85]$ \\
\hline \multirow{2}{*}{$x^{(4)}$} & {$[9.5,9.8$,} & {$[10,11$,} & {$[0.84,0.86$,} \\
& $10.3,10.7]$ & $12,13]$ & $0.87,0.9]$ \\
\hline
\end{tabular}

As a result, the following solutions were obtained, as shown in Table 5 .

Table 5

Optimization results

\begin{tabular}{|c|c|c|}
\hline $\begin{array}{c}\text { Alternatives to achieving } \\
\text { the goal of the operation }\end{array}$ & $\begin{array}{c}\text { Operation cost } \\
\text { vector }\end{array}$ & $\begin{array}{c}\text { Generalized } \\
\text { utility estimate }\end{array}$ \\
\hline$x^{(1)}$ & {$[600,656,661,721]$} & 0.94 \\
\hline$x^{(2)}$ & {$[600,692,710,736]$} & 0.72 \\
\hline$x^{(3)}$ & {$[600,674,693,709]$} & 0.55 \\
\hline$x^{(4)}$ & {$[600,685,743,750]$} & 0.69 \\
\hline
\end{tabular}

Table 2 shows that the alternative $x_{1}$ with the cost values [600, 656, 7661, 721] and the corresponding generalized utility $F\left(x^{(1)}\right)=0.94$ is the best alternative to applying grouping. The distribution vector $[600,656,7661,721]$ means that in this option of using the troops (forces) group, the implementation of the alternative $x^{(1)}$ to using the troops (forces) group will be from 656 to 717 million conventional units.

\section{Discussion of the results of estimation method development}

The main advantages of the proposed estimation method are:

- flexible hierarchical structure of indicators, which allows reducing the problem of multi-criteria evaluation of alternatives to one criterion or using the vector of indicators for selection - expressions (5)-(13);

- unambiguity of the obtained object state estimate - expressions (5)-(13);

- wide scope of use (decision support systems);

- no accumulation of training errors (Tables 2, 3);

- adaptability of the system of indicators - expressions (2)-(5);

- learning not only the synaptic weights of the artificial neural network, but also the type and parameters of the membership function (Tables 2, 3);

- learning the architecture of artificial neural networks (Tables 2, 3);

- data calculation for one epoch without the need to store previous calculations;

- consideration of the type of uncertainty while constructing a fuzzy cognitive temporal model (step 2 in Fig. 2 as a result of continuing [22]);

- the possibility of synthesizing the optimal structure of the decision support system - expressions (2)-(5); 
- the ability to quickly build a fuzzy cognitive temporal model by simultaneously searching for a solution by several individuals - expressions (5) - (13);

- the ability to avoid the problem of local extremum - expressions (5)-(13).

The disadvantages of the proposed method include:

- loss of informativeness in object state estimation due to the construction of the membership function. This loss of informativeness can be reduced by choosing the type of membership function in the practical implementation of the proposed method in decision support systems. The choice of the type of membership function depends on the computing resources of a particular computing device;

- lower estimation accuracy based on a separate estimation parameter of the object state;

- lower estimation accuracy compared to other estimation methods.

This method allows:

- estimating the state of the object;

- identifying effective measures to improve management efficiency;

- increasing the speed of object state estimation;

- reducing the use of computing resources of decision support systems.

According to the results of analyzing the effectiveness of the proposed method its computational complexity is 11-15\% less compared to other approaches for decision effectiveness estimation, presented in Table 2.

The proposed method can be used in decision support systems of automated control systems of artillery units, special-purpose geographic information systems. It can also be used in DSS for aviation and air defense ACS and DSS for logistics ACS of the Armed Forces.

The limitations of the study are the need for an initial object state database, the need to take into account the delay in collecting and proving information from intelligence sources.

This study is a further development of earlier research aimed at developing methodological principles for improving the efficiency of data processing in special-purpose information systems [2-6, 22, 24].

Further research should be aimed at reducing computational costs in processing various types of data in special-purpose information systems using artificial intelligence methods [25-34].

\section{Conclusions}

1. Mathematical description of the estimation process in intelligent decision support systems was carried out. This mathematical description allows describing the processes that take place in intelligent decision support systems when solving object state analysis problems. The efficiency of the object state analysis process is chosen as an efficiency criterion of this method. In this description, the analysis process is presented as a multidimensional time series. This allows creating a hierarchi- cal description of a complex process by levels of generalization and conducting appropriate analysis of its state.

2. The algorithm for implementing the method allows:

- conducting a multidimensional object state analysis under uncertainty;

- estimation under non-stochastic uncertainty, nonlinear interaction, partial inconsistency and significant interdependence of components of a multidimensional time series;

- performing a topological analysis of the NFCM structure;

- considering the effect of initial data noise on data processing efficiency;

- taking into account the delay in transferring information from extraction points to processing points;

- taking into account the initial type of uncertainty of initial data in NFCM construction;

- conducting structural and parametric training of artificial neural networks for intelligent decision support systems;

- performing quick construction of a fuzzy cognitive temporal model by simultaneously searching in several directions by several individuals.

3. An example of using the proposed method on the example of assessing the operational situation of the troops (forces) group is given. This example showed an increase in data processing efficiency at the level of $11-15 \%$ using additional advanced procedures.

\section{Acknowledgments}

The author's team expresses gratitude for assistance in preparing the paper to:

- Doctor of Technical Sciences, Professor Kuvshinov Oleksiy - Deputy Head of the Educational and Scientific Institute of the Ivan Chernyakhovsky National Defense University of Ukraine;

- Doctor of Technical Sciences, Senior Researcher Sova Oleg - Head of the Department of Automated Control Systems of the Military Institute of Telecommunications and Informatization named after Heroes of Kruty;

- Doctor of Technical Sciences, Senior Researcher Zhuravskiy Yuriy - Head of the Department of Electrical Engineering and Electronics of the Zhytomyr Military Institute named after S. P. Korolyov;

- Honored Worker of Science and Technology of Ukraine, Doctor of Technical Sciences, Professor Slyusar Vadym - Chief Researcher at the Central Research Institute of Armaments and Military Equipment of the Armed Forces of Ukraine;

- Doctor of Technical Sciences, Professor Rotshtein Oleksandr - Professor of the Mahon Lev Jerusalem Polytechnic Institute;

- Candidate of Technical Sciences, Associate Professor Bashkirov Oleksandr - Leading Researcher at the Central Research Institute of Armaments and Military Equipment of the Armed Forces of Ukraine.

\section{References}

1. Bashkyrov, O. M., Kostyna, O. M., Shyshatskyi, A. V. (2015). Rozvytok intehrovanykh system zviazku ta peredachi danykh dlia potreb Zbroinykh Ozbroiennia ta viyskova tekhnika, 1 (5), 35-39.

2. Dudnyk, V., Sinenko, Y., Matsyk, M., Demchenko, Y., Zhyvotovskyi, R., Repilo, I. et. al. (2020). Development of a method for training artificial neural networks for intelligent decision support systems. Eastern-European Journal of Enterprise Technologies, 3 (2 (105)), 37-47. doi: https://doi.org/10.15587/1729-4061.2020.203301 
3. Maistrenko, O., Khoma, V., Karavanov, O., Stetsiv, S., Shcherba, A. (2021). Devising a procedure for justifying the choice of reconnaissance-firing systems. Eastern-European Journal of Enterprise Technologies, 1 (3 (109)), 60-71. doi: https://doi.org/ 10.15587/1729-4061.2021.224324

4. Pievtsov, H., Turinskyi, O., Zhyvotovskyi, R., Sova, O., Zvieriev, O., Lanetskii, B., Shyshatskyi, A. (2020). Development of an advanced method of finding solutions for neuro-fuzzy expert systems of analysis of the radioelectronic situation. EUREKA: Physics and Engineering, 4, 78-89. doi: https://doi.org/10.21303/2461-4262.2020.001353

5. Zuiev, P., Zhyvotovskyi, R., Zvieriev, O., Hatsenko, S., Kuprii, V., Nakonechnyi, O. et. al. (2020). Development of complex methodology of processing heterogeneous data in intelligent decision support systems. Eastern-European Journal of Enterprise Technologies, 4 (9 (106)), 14-23. doi: https://doi.org/10.15587/1729-4061.2020.208554

6. Shyshatskyi, A., Zvieriev, O., Salnikova, O., Demchenko, Ye., Trotsko, O., Neroznak, Ye. (2020). Complex Methods of Processing Different Data in Intellectual Systems for Decision Support System. International Journal of Advanced Trends in Computer Science and Engineering, 9 (4), 5583-5590 doi: https://doi.org/10.30534/ijatcse/2020/206942020

7. Yeromina, N., Kurban, V., Mykus, S., Peredrii, O., Voloshchenko, O., Kosenko, V. et. al. (2021). The Creation of the Database for Mobile Robots Navigation under the Conditions of Flexible Change of Flight Assignment. International Journal of Emerging Technology and Advanced Engineering, 11 (05), 37-44. doi: https://doi.org/10.46338/ijetae0521_05

8. Petrosian, R., Chukhov, V., Petrosian, A. (2021). Development of a method for synthesis the FIR filters with a cascade structure based on genetic algorithm. Technology Audit and Production Reserves, 4 (2 (60)), 6-11. doi: https://doi.org/10.15587/2706-5448.2021.237271

9. Alpeeva, E. A., Volkova, I. I. (2019). The use of fuzzy cognitive maps in the development of an experimental model of automation of production accounting of material flows. Russian Journal of Industrial Economics, 12 (1), 97-106. doi: https://doi.org/ 10.17073/2072-1633-2019-1-97-106

10. Zagranovskaya, A. V., Eissner, Y. N. (2017). Simulation scenarios of the economic situation based on fuzzy cognitive maps. Modern economics: problems and solutions, 10 (94), 33-47. doi: https://doi.org/10.17308/meps.2017.10/1754

11. Simankov, V. S., Putyato, M. M. (2013). Issledovanie metodov kognitivnogo analiza. Sistemniy analiz, upravlenie i obrabotka informatsii, 13, 31-35.

12. Ko, Y.-C., Fujita, H. (2019). An evidential analytics for buried information in big data samples: Case study of semiconductor manufacturing. Information Sciences, 486, 190-203. doi: https://doi.org/10.1016/j.ins.2019.01.079

13. Ramaji, I. J., Memari, A. M. (2018). Interpretation of structural analytical models from the coordination view in building information models. Automation in Construction, 90,117-133. doi: https://doi.org/10.1016/j.autcon.2018.02.025

14. Pérez-González, C. J., Colebrook, M., Roda-García, J. L., Rosa-Remedios, C. B. (2019). Developing a data analytics platform to support decision making in emergency and security management. Expert Systems with Applications, 120, 167-184. doi: https:// doi.org/10.1016/j.eswa.2018.11.023

15. Chen, H. (2018). Evaluation of Personalized Service Level for Library Information Management Based on Fuzzy Analytic Hierarchy Process. Procedia Computer Science, 131, 952-958. doi: https://doi.org/10.1016/j.procs.2018.04.233

16. Chan, H. K., Sun, X., Chung, S.-H. (2019). When should fuzzy analytic hierarchy process be used instead of analytic hierarchy process? Decision Support Systems, 125, 113114. doi: https://doi.org/10.1016/j.dss.2019.113114

17. Osman, A. M. S. (2019). A novel big data analytics framework for smart cities. Future Generation Computer Systems, 91, 620-633. doi: https://doi.org/10.1016/j.future.2018.06.046

18. Gödri, I., Kardos, C., Pfeiffer, A., Váncza, J. (2019). Data analytics-based decision support workflow for high-mix low-volume production systems. CIRP Annals, 68 (1), 471-474. doi: https://doi.org/10.1016/j.cirp.2019.04.001

19. Harding, J. L. (2013). Data quality in the integration and analysis of data from multiple sources: some research challenges. The International Archives of the Photogrammetry, Remote Sensing and Spatial Information Sciences, XL-2/W1, 59-63. doi: https:// doi.org/10.5194/isprsarchives-xl-2-w1-59-2013

20. Papa, A., Shemet, Y., Yarovyi, A. (2021). Analysis of fuzzy logic methods for forecasting customer churn. Technology Audit and Production Reserves, 1 (2 (57)), 12-14. doi: https://doi.org/10.15587/2706-5448.2021.225285

21. Gorelova, G. V. (2013). Cognitive approach to simulation of large systems. Izvestiya YuFU. Tekhnicheskie nauki, 3, $239-250$.

22. Lutsenko, I., Fomovskaya, E., Oksanych, I., Koval, S., Serdiuk, O. (2017). Development of a verification method of estimated indicators for their use as an optimization criterion. Eastern-European Journal of Enterprise Technologies, 2 (4 (86)), 17-23. doi: https://doi.org/10.15587/1729-4061.2017.95914

23. Koshlan, A., Salnikova, O., Chekhovska, M., Zhyvotovskyi, R., Prokopenko, Y., Hurskyi, T. et. al. (2019). Development of an algorithm for complex processing of geospatial data in the special-purpose geoinformation system in conditions of diversity and uncertainty of data. Eastern-European Journal of Enterprise Technologies, 5 (9 (101)), 35-45. doi: https://doi.org/10.15587/ 1729-4061.2019.180197

24. Mahdi, Q. A., Shyshatskyi, A., Prokopenko, Y., Ivakhnenko, T., Kupriyenko, D., Golian, V. et. al. (2021). Development of estimation and forecasting method in intelligent decision support systems. Eastern-European Journal of Enterprise Technologies, 3 (9 (111)), 51-62. doi: https://doi.org/10.15587/1729-4061.2021.232718

25. Poloziuk, K., Yaremenko, V. (2020). Neural networks and Monte-Carlo method usage in multi-agent systems for sudoku problem solving. Technology Audit and Production Reserves, 6 (2 (56)), 38-41. doi: https://doi.org/10.15587/2706-5448.2020.218427 
26. Akanova, A., Kaldarova, M. (2020). Impact of the compilation method on determining the accuracy of the error loss in neural network learning. Technology Audit and Production Reserves, 6 (2 (56)), 34-37. doi: https://doi.org/10.15587/2706-5448.2020.217613

27. Leoshchenko, S., Oliinyk, A., Subbotin, S., Zaiko, T. (2020). Usage of swarm intelligence strategies during projection of parallel neuroevolution methods for neuromodel synthesis. Technology Audit and Production Reserves, 5 (2 (55)), 12-17. doi: https://doi.org/ 10.15587/2706-5448.2020.214769

28. Yaremenko, V., Syrotiuk, O. (2020). Development of a multi-agent system for solving domain dictionary construction problem. Technology Audit and Production Reserves, 4 (2 (54)), 27-30. doi: https://doi.org/10.15587/2706-5448.2020.208400

29. Lakhno, V., Sagun, A., Khaidurov, V., Panasko, E. (2020). Development of an intelligent subsystem for operating system incidents forecasting. Technology Audit and Production Reserves, 2 (2 (52)), 35-39. doi: https://doi.org/10.15587/2706-5448.2020.202498

30. Hoseini Alinodehi, S. P., Moshfe, S., Saber Zaeimian, M., Khoei, A., Hadidi, K. (2016). High-Speed General Purpose Genetic Algorithm Processor. IEEE Transactions on Cybernetics, 46 (7), 1551-1565. doi: https://doi.org/10.1109/tcyb.2015.2451595

31. Hou, N., He, F., Zhou, Y., Chen, Y., Yan, X. (2018). A Parallel Genetic Algorithm With Dispersion Correction for HW/SW Partitioning on Multi-Core CPU and Many-Core GPU. IEEE Access, 6, 883-898. doi: https://doi.org/10.1109/access.2017.2776295

32. Nobile, M. S., Cazzaniga, P., Besozzi, D., Colombo, R., Mauri, G., Pasi, G. (2018). Fuzzy Self-Tuning PSO: A settings-free algorithm for global optimization. Swarm and Evolutionary Computation, 39, 70-85. doi: https://doi.org/10.1016/j.swevo.2017.09.001

33. Nugroho, E. D., Wibowo, M. E., Pulungan, R. (2017). Parallel implementation of genetic algorithm for searching optimal parameters of artificial neural networks. 2017 3rd International Conference on Science and Technology - Computer (ICST). doi: https://doi.org/ 10.1109/icstc.2017.8011867

34. Bergel, A. (2020). Neuroevolution. Agile Artificial Intelligence in Pharo, 283-294. doi: https://doi.org/10.1007/978-1-4842-5384-7_14 